\title{
EFICIENCIA: EL CóDIGO gENÉtICO DE LA GESTIÓN
}

\author{
DANILO B. MACERA
}

- Profesor de Globalización: Apertura y Tendencias

Área Académica de Administración

Facultad de Negocios, UPC

\begin{abstract}
RESUMEN
Se analiza la relación entre los términos eficiencia, eficacia, y efectividad. Mientras que los dos primeros términos se refieren a los recursos y a los objetivos respectivamente, el tercero se refiere a la combinación de ambos. La eficiencia es un concepto que está presente en la naturaleza y dentro de ésta en el reino animal. Se presentan casos de empresas que han logrado un desempeño adecuado en términos de eficiencia. Las capacidades que se deben desarrollar para alcanzar eficiencia corresponden a estimar con precisión la cantidad óptima de producción de bienes y servicios; identificar los recursos estratégicos y gestionarlos; y por último, tener claridad en el establecimiento de objetivos.
\end{abstract}

Palabras clave: Eficiencia, eficacia, efectividad.

\section{ABSTRACT}

This essay analyses the relationship between efficiency, efficacy, and effectivity. While the first two concepts refer to resources and objectives, respectively, the third one is related to the combination of both concepts. Efficiency is a concept present in nature, specifically in the animal kingdom. This essay presents success cases of companies that have archived efficiency; and identifies three capabilities that any organization must develop to reach efficiency, these being the optimal estimation of production quantity of good and services; identification of strategic resources and their management; and, finally, the determination of objectives with clarity.

Keywords: Efficiency, efficacy, effectiveness.

La batalla de Berlín se desarrolló en la primavera de 1945. Este sería el último episodio sangriento de grandes proporciones en el frente europeo durante la Segunda Guerra Mundial. Para la defensa de la Alemania nazi, el eje desplegó aproximadamente un total de 766700 soldados, 1500 tanques, 9300 cañones y 2200 aviones. Por su parte, el Komintern asignó a un aproximado de 2500000 soldados - entre soviéticos y polacos-, con un material de 6200 tanques, 41600 cañones y 7500 aviones (Ziemke, 1969). Es decir, las fuerzas soviéticas y - sin contar a sus aliados Estados Unidos, Gran Bretaña y Francia-casi cuadriplicaban a las alemanas. Tras lanzarse aproximadamente siete millones de proyectiles y realizarse más de 6500 incursiones aéreas, el 30 de abril Adolf Hitler se suicidaría, iniciándose así el comienzo de la derrota alemana. Un dato curioso es que el Komintern sufrió 361300 bajas, entre 81100 muertos y 280200 heridos, mientras que el eje tuvo 280000 , entre 70000 muertos y 220000 heridos. Es decir, las pérdidas soviéticas superaron en 30\% a las alemanas. En cuanto a las pérdidas alemanas, estas representaban alrededor del $8 \%$ de las fuerzas en combate, mientras que las del ejército rojo superaron el 11\%. Para muchos analistas militares, las fuerzas desplegadas por Stalin mostraron un uso desbordado de recursos, y la mayor exposición de recursos devino en una mayor pérdida de vidas.

Aunque el logro del objetivo es vital, debe ir de la mano con el uso óptimo de los recursos; si no, estamos frente a una acción torpe y carente de gestión. Los líderes y directivos tienen la obligación de hacer una gestión que alcance los objetivos de la organización, pero esta debe hacerse considerando la limitación de recursos y su uso óptimo, que conlleve a alcanzar altos niveles de eficiencia y de retorno sobre los recursos invertidos.

El libro The Effective Executive (o El ejecutivo eficaz), publicado hace cinco décadas por el austriaco Peter Drucker -y, en términos más amplios, toda su vasta producción-, ahonda en la esencia misma de la gestión administrativa, pues sus páginas discurren a través de conceptos fundamentales de esta: eficiencia, "hacer bien las cosas", es el cómo: evidencia la relación óptima entre recursos y resultados; eficacia, "hacer las cosas correctas", es el qué: se orienta exclusivamente al logro de objetivos: efectividad, el tercer término usado por Drucker, vincula a los dos primeros: "hacer bien las cosas correctas". Este término reclama tanto eficiencia como eficacia: el qué y el cómo. Sin embargo, la efectividad, como tercer término, sale sobrando para muchos autores, dado que en la eficiencia se da por sentado el logro del objetivo. Por ello, para todo fin usaremos este último, susceptible de una más amplia cobertura verbal y que se diferencia con mayor claridad de la pura consecución de la meta - eficacia - en un sentido que hace referencia tanto al logro como al uso óptimo de recursos.

Si pensamos en eficiencia, la naturaleza es, sin duda, la máxima gestora de esta. Solemos equivocadamente llamar indistintamente pompas y burbujas a las 
superficies que se forman con el jabón; lo cierto es que las primeras - pompas - no retienen aire en su interior, mientras que las burbujas sí lo hacen. Y, por supuesto, todos sabemos qué forma tiene una burbuja de jabón: la de esfera. La pregunta es la siguiente: ¿por qué esa y no otra figura? Sin esquinas y completamente simétrica. Esto se debe a que es la forma que economiza mayor cantidad de material, y por tanto es la figura más eficiente. Los planetas, la Luna, tienen esta forma. ¿Y qué ocurre cuándo dos o más burbujas de jabón se encuentran? Pues comparten la superficie de contacto economizando material, y su geometría se altera. Así, cuatro burbujas buscarán la forma más eficiente y compartirán un tetraedro (cuatro caras triangulares); y, si se incorporan dos más, tendríamos un cubo; doce burbujas formarán un dodecaedro y así sucesivamente, economizando energía y espacio. Pappus de Alejandría, matemático griego de los siglos III y IV, demostró que, entre todos los polígonos regulares con el mismo perímetro, encierran más área aquellos que tienen mayor cantidad de lados. Por eso, la figura que encierra mayor área para un perímetro determinado es el círculo, que en su proyección tridimensional es una esfera, la misma que posee un número infinito de lados. La naturaleza es perezosa. Bill Gates suele decir: "Prefiero una persona perezosa para hacer un trabajo duro, porque una persona perezosa encontrará una manera sencilla de hacerlo".

Una de las evidencias más sorprendentes de eficiencia en la naturaleza es, sin duda, la construcción de un panal de abejas. Este panal ha sido hecho íntegramente de cera, y es tan costoso producir este material que las abejas deben ser altamente eficientes. Un solo kilogramo de cera requiere que las abejas vuelen el equivalente a veinticuatro vueltas a la Tierra. Con una vida media de 85 días y ante recursos limitados, han de buscar el modo más eficiente, que conceda más espacio y economice material. Como sabemos, los panales de abejas forman un mosaico, embaldosado o tesela, donde concurren hexágonos perfectos con ángulos de $120^{\circ}$, pero existen otras formas posibles que no dejan espacio entre ellas, como el cuadrado o el triángulo equilátero, esto debido a que los ángulos que forman sus lados, al igual que los del hexágono, son divisores enteros de $360^{\circ}: 60^{\circ}$ para el triángulo equilátero, $90^{\circ}$ para el cuadrado y $120^{\circ}$ para el hexágono. (Botanical-online, s.f.). Pero, entonces, ¿por qué hacer hexágonos y no las otras formas más sencillas? Pues, a partir de la demostración de Pappus o de las observaciones de Charles Darwin, se vio que el hexágono es más eficiente que el triángulo o el cuadrado por su mayor numero de lados. Esta norma está codificada en el ADN de las abejas, lo que conlleva a una gestión exitosa y a un uso racional de recursos.

Tal vez en algún momento existieron diferentes colonias de abejas que optaron por panales con un embaldosado triangular periódico; u otras, las que prefirieron los cuadrados o incluso los círculos y pentágonos, o combinaciones de estos - teselas no periódicas. Y, por supuesto, las que eligieron el hexágono. Al igual que las naciones y las empresas, prevalecieron aquellas que optimizaron el uso de los diversos pero no ilimitados recursos: tiempo, materiales, espacio, horas de trabajo, etcétera. Y son estas las que hoy determinan un modelo, así como en su momento ocurrió con las grandes industrias que abrazaron el cambio y no lucharon contra él, tras la Revolución Industrial.

En el año 2013, la tienda por departamentos chilena Saga Falabella tuvo ventas por USD MM 953,70 en el Perú, con una inversión en marketing de USD MM 23,60; es decir, su ratio de ventas sobre inversión en marketing para dicho periodo indica que, por cada unidad monetaria destinada al marketing de la empresa, se lograron 40,41 unidades monetarias de venta. Sin embargo, su competidor y connacional Ripley, que le siguió en nivel de ventas en esta categoría con USD MM 936,30, destinó a marketing en dicho periodo menos del $60 \%$ de lo hecho por Saga Falabella: USD MM 13,90. Por cada unidad monetaria empleada en marketing, Ripley generó 67,36 unidades monetaria en ventas, alrededor de 27 unidades monetarias más. Este es un claro indicador de eficiencia y de la gestión exitosa de recursos.

El AD Alcorcón de la segunda división del fútbol español humilló al poderoso Real Madrid de Raúl y Cristiano Ronaldo - entre otras estrellas-, al golearlo por cuatro a cero en el partido de ida de los dieciseisavos de final de la Copa del Rey de 2009. Precisamente ese año, el Real Madrid había pagado 96 millones de euros por su máxima estrella, Cristiano Ronaldo. En la actualidad el Real Madrid, con una plantilla de estrellas que ronda los 700 millones de euros, encabeza en valor económico el ranking mundial en este deporte; El Real Madrid, que ese año tenía un presupuesto de 400 millones, no pudo anotarle un solo gol al modesto representante de la ciudad de Alcorcón, cuyo presupuesto llegaba apenas a un millón de euros. Es decir, y en este contexto, el Real Madrid "invirtió" 100 millones de euros por gol recibido; y el Alcorcón, por su parte, destinó cuatrocientas veces menos para anotar cada uno de sus cuatro goles en el arco del gigante ibérico.

Es bastante claro que, ya sea en una colmena de abejas, en una tienda por departamentos, en la guerra o en el deporte, la gestión óptima de los recursos acredita el éxito. Por supuesto, los recursos difieren: la cera de las abejas, la publicidad de los almacenes, las armas de guerra o la habilidad futbolística; pero el medio que faculta el logro se basa en la gestión eficiente de estos recursos. Es importante subrayar que un exceso de asignación de los mismos, como en los casos de Stalin o del Real Madrid, es tan peligroso como su falta. Por tanto, se espera una cantidad óptima. El Diccionario de la lengua española incluye este adjetivo bajo el significado de "sumamente bueno, que no puede ser mejor". Así, debemos desarrollar la capacidad de estimar con la máxima precisión posible esta cantidad óptima. De esa manera, no arriesguemos la consecución del objetivo por falta de recursos, pero tampoco afectemos el retorno de la inversión por el exceso irresponsable en la asignación de los mismos.

Por su parte, es evidente que el marketing no fue el único recurso que determinó el éxito en ventas de Ripley, así como tampoco lo fue la habilidad futbolística del Alcorcón, pero sin duda estos aspectos fueron determinantes. De tal forma, otra capacidad crítica en la gestión sería la de identificar y 
discriminar los recursos estratégicos de aquellos que no son tales, y, en consecuencia, protegerlos y potenciarlos; es decir: gestionarlos al más alto nivel de desempeño.

La exitosa cadena española Zara, con presencia en cinco continentes (Europa, América, África, Asia y Oceanía), con más de 7000 locales en 88 países y ventas que superan los veinte mil millones de euros, no utiliza el 100\% de su capacidad instalada en la producción de sus prendas; es más, esta tienda de moda, perteneciente al grupo Inditex y fundada por Amancio Ortega, trabaja muy por debajo de este porte. Entonces, ¿no es eficiente Zara?

La tercera capacidad que debe desarrollar un directivo es la claridad de objetivos. Toda gestión debe estar orientada a un propósito específico. Zara basa su éxito comercial en gran medida en la administración impecable de su cadena de suministro. El uso aparentemente ineficiente de la capacidad de su planta de producción le permite asegurar la eficiencia de su sistema de abastecimiento global, atendiendo así distintas necesidades por volumen de demanda, gustos e intereses, variables culturales y diferentes temporadas en casi una centena de países simultáneamente. Tal vez, a la luz de esta habilidad, alguien pudiera sugerir que el Real Madrid tenía objetivos diferentes de los del AD Alcorcón, o que la eficiencia no se mide en una sola jornada. Lo cierto es que el multimillonario equipo se quedó en dicha etapa (dieciseisavos de final) y el AD Alcorcón de segunda división avanzó hasta octavos de final en la competencia.

Tal vez la famosa frase de Winston Churchill, "nunca tantos debieron tanto a tan pocos", que fue pronunciada como tributo a la estrategia del mariscal en jefe del aire Hugh Dowding, quien dirigió el Mando de Caza de la Royal Air Force durante la batalla de Inglaterra, a principios de la Segunda Guerra Mundial, sea un reconocimiento oficial a la eficiencia y, en consecuencia, a la correcta identificación de recursos estratégicos, a la asignación óptima de estos, a la claridad de objetivos y a su consecuente adquisición exitosa.

\section{REFERENCIAS}

Botanical-online. (s.f.). La cera de abeja. Recuperado de https://www.botanical-online.com/ceradeabeja.htm

Drucker, P. (2014). El ejecutivo eficaz. Argentina: Penguin Random House Grupo Editorial.

Ziemke, E., F. (1969). Battle for Berlin End of the Third Reich,Ballantine's Illustrated History of World War II (Battle Book \#6). New York, NY: Ballantine Books. 